\title{
ON A SINGULAR PERTURBATION PROBLEM FOR LINEAR SYSTEMS OF ORDINARY DIFFERENTIAL EQUATIONS, I
}

\author{
By YoshikazU HiRAsawa
}

1. In a paper [1], we had to treat the following singular perturbation problem.

Let us consider two linear systems of ordinary differential equations containing a small positive parameter $\varepsilon$ :

$$
\begin{aligned}
& \varepsilon \frac{d \boldsymbol{u}}{d t}=\left(A_{0}-\varepsilon A_{1}\right) \boldsymbol{u}+\boldsymbol{\delta}_{1}(\varepsilon), \\
& \varepsilon \frac{d \boldsymbol{u}}{d t}=\left(A_{0}+\varepsilon A_{1}\right) \boldsymbol{u}+\boldsymbol{\delta}_{2}(\varepsilon),
\end{aligned}
$$

where

$$
\boldsymbol{u}=\left(\begin{array}{l}
u_{1} \\
u_{2} \\
u_{3}
\end{array}\right), \quad A_{0}=\left(\begin{array}{rrr}
0 & 0 & 0 \\
\alpha & -\beta & \gamma \\
-\alpha & -\gamma & \beta
\end{array}\right), \quad A_{1}=\left(\begin{array}{lll}
\alpha & \alpha & \alpha \\
0 & 0 & 0 \\
0 & 0 & 0
\end{array}\right) \text {, }
$$

and $\alpha, \beta, \gamma$ are positive constants such that $\gamma<\beta$.

Further,

$$
\boldsymbol{\delta}_{1}(\varepsilon)=\left(\begin{array}{r}
-\varepsilon \delta_{1}(\varepsilon) \\
\delta_{2}(\varepsilon) \\
\delta_{3}(\varepsilon)
\end{array}\right), \quad \boldsymbol{\delta}_{2}(\varepsilon)=\left(\begin{array}{c}
\varepsilon \delta_{1}(\varepsilon) \\
\delta_{2}(\varepsilon) \\
\delta_{3}(\varepsilon)
\end{array}\right),
$$

and $\delta_{j}(\varepsilon) \rightarrow 0(\jmath=1,2,3)$ for $\varepsilon \rightarrow+0$.

Given an interval $t_{1} \leqq t \leqq t_{2}$ and a point $t_{0}$ such that $t_{1} \leqq t_{0} \leqq t_{2}$, we need a set of continuous functions $u_{1}(t ; \varepsilon), u_{2}(t ; \varepsilon), u_{3}(t ; \varepsilon)$ with the following properties.

( I ) The conditions

$$
u_{1}\left(t_{0} ; \varepsilon\right)=P(\varepsilon), \quad u_{2}\left(t_{1} ; \varepsilon\right)=Q(\varepsilon), \quad u_{3}\left(t_{2} ; \varepsilon\right)=R(\varepsilon)
$$

are fulfilled, where $P(\varepsilon), Q(\varepsilon)$ and $R(\varepsilon)$ are suitable positive quantities tending to zero with $\varepsilon$, such that $P_{0}(\varepsilon) \leqq P(\varepsilon), Q_{0}(\varepsilon) \leqq Q(\varepsilon), R_{0}(\varepsilon) \leqq R(\varepsilon)$ for given positive quantities $P_{0}(\varepsilon), Q_{0}(\varepsilon), R_{0}(\varepsilon)$ tending to zero with $\varepsilon$.

(II) $u_{1}(t ; \varepsilon), u_{2}(t ; \varepsilon), u_{3}(t ; \varepsilon)$ are positive on $t_{1} \leqq t \leqq t_{2}$.

Recerved August 29, 1976 
(III) $\boldsymbol{u}=\boldsymbol{u}(t ; \varepsilon)$ satisfies the system (1) for $t_{1} \leqq t \leqq t_{0}$ and satisfies the system (2) for $t_{0} \leqq t \leqq t_{2}$.

In the paper [1], we gave a solution of the above problem, but the proof was not complete.

In this paper, we will give a new proof of the existence of such a solution rather in a special case. That is, we take the case $t_{0}=t_{1}$ and hence we omit the system (1).

2. Put

$$
A=\frac{A_{0}}{\varepsilon}+A_{1}=\left(\begin{array}{rrr}
\alpha & \alpha & \alpha \\
\frac{\alpha}{\varepsilon} & -\frac{\beta}{\varepsilon} & \frac{\gamma}{\varepsilon} \\
-\frac{\alpha}{\varepsilon} & -\frac{\gamma}{\varepsilon} & \frac{\beta}{\varepsilon}
\end{array}\right),
$$

then the characteristic equation of $A$ is

$$
\varepsilon^{2} \lambda^{3}-\varepsilon^{2} \alpha \lambda^{2}-\left(\beta^{2}-\gamma^{2}\right) \lambda+\alpha \beta^{2}-\alpha \gamma^{2}+2 \alpha^{2} \beta+2 \alpha^{2} \gamma=0 .
$$

Since the roots $\rho_{1}, \rho_{2}, \rho_{3}$ of this equation can be regarded as algebraic functions of $\varepsilon$, we put

or

$$
\lambda=a_{0}+a_{1} \varepsilon+\cdots,
$$

$$
\lambda=\frac{b_{-1}}{\varepsilon}+b_{0}+b_{1} \varepsilon+\cdots,
$$

to the end of finding these roots.

Substituting these series into (4) and determining the coefficients $a_{0}, a_{1}, \cdots$, or $b_{-1}, b_{0}, \cdots$, as the characteristic roots $\rho_{1}, \rho_{2}, \rho_{3}$ of $A$, we get

$$
\begin{aligned}
& \rho_{1}=\frac{2 \alpha^{2}+\alpha(\beta-\gamma)}{\beta-\gamma}+O(\varepsilon), \\
& \rho_{2}=-\frac{\mu}{\varepsilon}+O(1), \quad \rho_{3}=\frac{\mu}{\varepsilon}+O(1),
\end{aligned}
$$

where $\mu=\sqrt{\beta^{2}-\gamma^{2}}$.

Furthermore the canonical form $\hat{A}$ of $A$ is

$$
\hat{A}=\left(\begin{array}{ccc}
\rho_{1} & & 0 \\
& \rho_{2} & \\
0 & & \rho_{3}
\end{array}\right)
$$

and the transformation matrix $S(\varepsilon)$ that transforms $A$ into $\hat{A}$ is 
(6)

$$
\begin{aligned}
S(\varepsilon) & =\left(s_{i j}(\varepsilon)\right) \\
& =\left(\begin{array}{ccc}
\beta-\gamma+O(\varepsilon) & O(\varepsilon) & O(\varepsilon) \\
\alpha+O(\varepsilon) & \gamma+O(\varepsilon) & \gamma+O(\varepsilon) \\
\alpha+O(\varepsilon) & \beta-\sqrt{\beta^{2}-\gamma^{2}}+O(\varepsilon) & \beta+\sqrt{\beta^{2}-\gamma^{2}}+O(\varepsilon)
\end{array}\right) .
\end{aligned}
$$

That is, $S(\varepsilon)^{-1} A S(\varepsilon)=\hat{A}$.

By the transformation of unknowns $\boldsymbol{u}=S(\varepsilon) \boldsymbol{v}$, the system (2) is changed into

$$
\frac{d \boldsymbol{v}}{d t}=\hat{A} \boldsymbol{v}+\frac{1}{\varepsilon} \hat{\boldsymbol{\delta}}_{2}(\varepsilon), \quad \hat{\boldsymbol{\delta}}_{2}(\varepsilon)=S(\varepsilon)^{-1} \boldsymbol{\delta}_{2}(\varepsilon) .
$$

Now, let

$$
\boldsymbol{\omega}(\varepsilon)=\left(\begin{array}{c}
\omega_{1}(\varepsilon) \\
\omega_{2}(\varepsilon) \\
\omega_{3}(\varepsilon)
\end{array}\right)
$$

be a solution of a linear equation

$$
\left(A_{0}+\varepsilon A_{1}\right) \boldsymbol{\omega}+\boldsymbol{\delta}_{2}(\varepsilon)=\mathbf{0} .
$$

Then, clearly $\omega_{j}(\varepsilon) \rightarrow 0(\jmath=1,2,3)$ for $\varepsilon \rightarrow+0$.

We will seek for a desired solution in the following form:

$$
\boldsymbol{u}(t ; \varepsilon)=\left(\begin{array}{c}
u_{1}(t ; \varepsilon) \\
u_{2}(t ; \varepsilon) \\
u_{3}(t ; \varepsilon)
\end{array}\right)=S(\varepsilon)\left(\begin{array}{c}
C_{1}(\varepsilon) e^{\rho_{1}\left(t-t_{1}\right)} \\
C_{2}(\varepsilon) e^{\rho_{2}\left(t-t_{1}\right)} \\
C_{3}(\varepsilon) e^{\rho_{3}\left(t-t_{2}\right)}
\end{array}\right)+\left(\begin{array}{c}
\omega_{1}(\varepsilon) \\
\omega_{2}(\varepsilon) \\
\omega_{3}(\varepsilon)
\end{array}\right)
$$

3. It is sufficient to determine the positive quantities $P(\varepsilon), Q(\varepsilon), R(\varepsilon)$ and the coefficients $C_{j}(\varepsilon)(\jmath=1,2,3)$ so that

$$
\left\{\begin{array}{l}
C_{1}(\varepsilon) s_{11}(\varepsilon)+C_{2}(\varepsilon) s_{12}(\varepsilon)+C_{3}(\varepsilon) s_{13}(\varepsilon) e^{\rho_{3}\left(t_{1}-t_{2}\right)}=\hat{P}(\varepsilon)\left(=P(\varepsilon)-\omega_{1}(\varepsilon)\right), \\
C_{1}(\varepsilon) s_{21}(\varepsilon)+C_{2}(\varepsilon) s_{22}(\varepsilon)+C_{3}(\varepsilon) s_{23}(\varepsilon) e^{\rho_{3}\left(t_{1}-t_{2}\right)}=\hat{Q}(\varepsilon)\left(=Q(\varepsilon)-\omega_{2}(\varepsilon)\right), \\
C_{1}(\varepsilon) s_{31}(\varepsilon) e^{\rho_{1}\left(t_{2}-t_{1}\right)}+C_{2}(\varepsilon) s_{32}(\varepsilon) e^{\rho_{2}\left(t_{2}-t_{1}\right)}+C_{3}(\varepsilon) s_{33}(\varepsilon)=\hat{R}(\varepsilon)\left(=R(\varepsilon)-\omega_{3}(\varepsilon)\right),
\end{array}\right.
$$

and $u_{1}(t ; \varepsilon), u_{2}(t ; \varepsilon), u_{3}(t ; \varepsilon)$ are positive on the interval $t_{1} \leqq t \leqq t_{2}$.

By virtue of $\rho_{2}<0, \rho_{3}>0$, we see easily

$$
\begin{aligned}
\Delta(\varepsilon) & =\left|\begin{array}{lll}
s_{11}(\varepsilon) & s_{12}(\varepsilon) & s_{13}(\varepsilon) e^{\rho_{3}\left(t_{1}-t_{2}\right)} \\
s_{21}(\varepsilon) & s_{22}(\varepsilon) & s_{23}(\varepsilon) e^{\rho_{3}\left(t_{1}-t_{2}\right)} \\
s_{31}(\varepsilon) e^{\rho_{1}\left(t_{2}-t_{1}\right)} & s_{32}(\varepsilon) e^{\rho_{2}\left(t_{2}-t_{1}\right)} & s_{33}(\varepsilon)
\end{array}\right| \\
& =(\beta-\gamma) \gamma\left(\beta+\sqrt{\beta^{2}-\gamma^{2}}\right)+O(\varepsilon),
\end{aligned}
$$




$$
\begin{aligned}
& \Delta_{1}(\varepsilon)=\left|\begin{array}{ccc}
\hat{P}(\varepsilon) & s_{12}(\varepsilon) & s_{13}(\varepsilon) e^{\rho_{3}\left(t_{1}-t_{2}\right)} \\
\hat{Q}(\varepsilon) & s_{22}(\varepsilon) & s_{23}(\varepsilon) e^{\rho_{3}\left(t_{1}-t_{2}\right)} \\
\hat{R}(\varepsilon) & s_{32}(\varepsilon) e^{\rho_{2}\left(t_{2}-t_{1}\right)} & s_{33}(\varepsilon)
\end{array}\right| \\
& =\gamma\left(\beta+\sqrt{\beta^{2}-\gamma^{2}}\right) \hat{P}(\varepsilon)+O(\varepsilon), \\
& \Delta_{2}(\varepsilon)=\left|\begin{array}{ccc}
s_{11}(\varepsilon) & \hat{P}(\varepsilon) & s_{13}(\varepsilon) e^{\rho_{3}\left(t_{1}-t_{2}\right)} \\
s_{21}(\varepsilon) & \hat{Q}(\varepsilon) & s_{23}(\varepsilon) e^{\rho_{3}\left(t_{1}-t_{2}\right)} \\
s_{31}(\varepsilon) e^{\rho_{1}\left(t_{2}-t_{1}\right)} & \hat{R}(\varepsilon) & s_{33}(\varepsilon)
\end{array}\right| \\
& =\left(\beta+\sqrt{\beta^{2}-\gamma^{2}}\right)\{-\alpha \hat{P}(\varepsilon)+(\beta-\gamma) \hat{Q}(\varepsilon)\}+O(\varepsilon) \text {, } \\
& \Delta_{3}(\varepsilon)=\left|\begin{array}{clc}
s_{11}(\varepsilon) & s_{12}(\varepsilon) & \hat{P}(\varepsilon) \\
s_{21}(\varepsilon) & s_{22}(\varepsilon) & \hat{Q}(\varepsilon) \\
s_{31}(\varepsilon) e^{\rho_{1}\left(t_{2}-t_{1}\right)} & s_{32}(\varepsilon) e^{\rho_{2}\left(t_{2}-t_{1}\right)} & \hat{R}(\varepsilon)
\end{array}\right| \\
& =\gamma\left\{-\alpha e^{\rho_{1}\left(t_{2}-t_{1}\right)} \hat{P}(\varepsilon)+(\beta-\gamma) \hat{R}(\varepsilon)\right\}+O(\varepsilon) .
\end{aligned}
$$

Hence we have

$$
\begin{aligned}
& C_{1}(\varepsilon)=\frac{1}{\beta-\gamma} \hat{P}(\varepsilon)+O(\varepsilon), \\
& C_{2}(\varepsilon)=\frac{1}{\gamma(\beta-\gamma)}\{-\alpha \hat{P}(\varepsilon)+(\beta-\gamma) \hat{Q}(\varepsilon)\}+O(\varepsilon), \\
& C_{3}(\varepsilon)=\frac{1}{(\beta-\gamma)\left(\beta+\sqrt{\left.\beta^{2}-\gamma^{2}\right)}\right.}\left\{-\alpha e^{\rho_{1}\left(t_{2}-t_{1}\right)} \hat{P}(\varepsilon)+(\beta-\gamma) \hat{R}(\varepsilon)\right\}+O(\varepsilon) .
\end{aligned}
$$

This shows that we can take

$$
\begin{aligned}
& P(\varepsilon)=\left(\hat{P}(\varepsilon)+\omega_{1}(\varepsilon)\right), \\
& Q(\varepsilon)=\left(\hat{Q}(\varepsilon)+\omega_{2}(\varepsilon)\right), \\
& R(\varepsilon)=\left(\hat{R}(\varepsilon)+\omega_{3}(\varepsilon)\right),
\end{aligned}
$$

such that $C_{j}(\varepsilon)>0(\jmath=1,2,3)$, and $u_{\jmath}(t ; \varepsilon)>0(\jmath=1,2,3)$ on the interval $t_{1} \leqq t \leqq t_{2}$.

\section{REFERENCE}

[1] Y. Hirasawa, On singular perturbation problems of non-linear systems of differential equations, III, Comment. Math. Univ. Sancti. Pauli, 4 (1955), 93-104.

Department of MATHEMAtics,

Tokyo Institute of Technology 